\title{
Le cycle de la vie familiale au Québec : vues comparatives, $\mathrm{XVII}^{\mathrm{e}}-\mathrm{XX}^{\mathrm{e}}$ siècles
}

The Family Life Cycle in Quebec: Comparative Views, 17th-20th

Centuries

\section{El ciclo de vida de las familias de Quebec: examen comparativo, siglos XVII y XX}

\author{
Évelyne Lapierre-Adamcyk, Yves Landry, Jacques Légaré, Denis Morissette et
}

Yves Péron

Volume 13, numéro 1, avril 1984

Population et histoire

URI : https://id.erudit.org/iderudit/600521ar

DOI : https://doi.org/10.7202/600521ar

Aller au sommaire du numéro

Éditeur(s)

Association des démographes du Québec

ISSN

0380-1721 (imprimé)

1705-1495 (numérique)

Découvrir la revue

Citer cet article

Lapierre-Adamcyk, É., Landry, Y., Légaré, J., Morissette, D. \& Péron, Y. (1984). Le cycle de la vie familiale au Québec : vues comparatives, $\mathrm{XVII}^{\mathrm{e}}-\mathrm{XX}^{\mathrm{e}}$ siècles. Cahiers québécois de démographie, 13(1), 59-77.

https://doi.org/10.7202/600521ar

\section{Résumé de l'article}

Cet article vise à comparer le cycle de la vie familiale tel qu'on a pu l'observer au Québec à deux époques éloignées l'une de l'autre : la fin du XVII ${ }^{\mathrm{e}}$ siècle et le milieu du $\mathrm{XX}^{\mathrm{e}}$ siècle. Le calcul d'indices comparables a été réalisé pour les deux périodes en l'absence d'événements perturbateurs. Il permet de faire ressortir les traits marquants de l'évolution du cycle de la vie familiale pour les familles qui en traversaient toutes les phases ; soulignons en particulier la réduction considérable de la durée de la phase d'agrandissement, liée à la très forte baisse de la fécondité, et l'apparition d'une longue phase de stabilisation réelle au cours de laquelle les parents ont charge de tous leurs enfants. Un rapprochement avec la proportion des faillies que représentent les unions non rompues laisse enfin apparaître de façon éclatante l'impact de la mortalité sur la vie familiale d'autrefois.
Tous droits réservés @ Association des démographes du Québec, 1984

Ce document est protégé par la loi sur le droit d'auteur. L'utilisation des services d'Érudit (y compris la reproduction) est assujettie à sa politique d'utilisation que vous pouvez consulter en ligne.

https://apropos.erudit.org/fr/usagers/politique-dutilisation/ 
Cahiers québécois de démographie

Vol. 13, no 1 , avril 1984

\title{
Le cycle de la vie familiale au Québec: vues comparatives, XVIle-XXe siecles
}

\author{
Évelyne LAPIERRE-ADAMCYK, Yves LANDRY, \\ Jacques LÉGARÉ, Denis MORISSETTE et Yves PÉRON*
}

\section{INTRODUCTION}

La notion de cycle de vie a d'abord éte utilisée en demographie pour illustrer l'enchaînement des divers evénements démographiques qui marquent la vie des individus. C'est ainsi que, par exemple, Fourastié (1959) a etabli le calendrier demographique de l'homme moyen sur la base d'informations relatives au début du XVIIIe siécle et l'a comparé a celui de l'homme moderne. Livi-Bacci (1978), dans le meme esprit, s'est attaché aux événements liés a la constitution de la descendance, l'age moyen de la femme lui servant de repere. Cette notion de cycle de vie peut tout aussi bien s'appliquer au déroulement du cycle de vie des familles. Glick et Parke (1965) l'ont utilisée pour étudier les familles américaines du XXe siecle. Leurs travaux sont d'ailleurs a l'origine de la popularité dont jouit depuis quelques annés la recherche sur le cycle de la vie familiale. Les concepts sur lesquels elle repose, quoique deja mis en question par les brusques changements qui affectent la famille depuis la derniere décennie, continuent doffrir un cadre extremement intéressant et stimulant pour comprendre l'impact des transformations des phénomènes démographiques sur la famille, cellule de base des societés.

Cet article vise a comparer le cycle de la vie familiale tel qu'on a pu l'observer au Quebec à deux époques éloignées l'une de l'autre: la fin du XVIIe siecle et le milieu du XXe siecle. Un article de Landry et Legaré (1984) a déja présenté de façon détaillée le cycle de la vie familiale des unions formés avant 1700 en insistant sur les différences dans le déroulement du cycle selon quelques caractéristiques du couple, dont l'écart d'age entre conjoints, l'age au mariage ainsi que le lieu de naissance. Les cohortes de la période moderne, par ailleurs, ont eté étudiees dans le cadre diune

* Groupe de recherche sur la démographie québecoise, Departement de démographie, Université de Montréal.

Cette recherche a été réalisée grace aux subventions du Conseil de recherches en sciences humaines du Canada (498-83-0008, 411-82-0004), du Fonds FCAC (84-CE-126, 84-EQ-2337) et de l'Université de Montréal. Les auteurs tiennent a remercier Michel Chouinard et Paul-Marie Huot pour leur contribution a la préparation des données. 
recherche qui vise essentiellement a développer une méthodologie pour classer les familles selon le stade atteint dans le cycle de la vie familiale au moment d'un recensement et de mesurer, de façon approximative, le calendrier des dernieres phases du cycle (Péron et Lapierre-Adamcyk, 1984). Pour les fins de la comparaison entre le passé lointain et la periode moderne, nous avons retenu la démarche suivante. Sur la base d'une définition assez souple des phases du cycle de la vie familiale, le calcul d'indices comparables a été réalise pour les deux periodes. Ces indices, des. medianes, des moyennes ou des proportions, ont été établis en l'absence d'événements perturbateurs; un rapprochement avec la proportion des familles que représentent les unions non rompues fait enfin ressortir de façon éclatante l'impact de la mortalité sur la vie familiale d'autrefois.

\section{DÉFINITION DU CYCLE DE LA VIE FAMILIALE}

On trouve a la figure 1 une illustration schématique des diverses phases du cycle de la vie familiale jalonnés par les évenements qui marquent le début et la fin de chacune d'entre elles. Trois grandes phases constituent l'ensemble du cycle. Il s'agit de:

- la phase préparentale qui va de la formation du couple par le mariage jusqu'a la naissance du premier enfant;

- la phase parentale dont la durée s'étend de la premiere naissance jusqu'au mariage ou au départ du dernier enfant; cette période se scinde elle-méme en deux sequences: celle de l'agrandissement/contraction, qui va de la premiere a la derniere naissance; celle de la stabilisation/contraction qui s'étend de la derniere naissance jusquau mariage ou au depart du dernier enfant; la contraction qui peut se produire a tout moment par le decess diun des enfants résulte aussi du mariage ou du départ de chaque enfant;

- la phase post-parentale qui s'amorce avec le mariage ou le depart du dernier enfant pour se terminer par la dissolution de la famille; elle est caracterisée par une premiere période où les deux conjoints qui ont fondé la fanille survivent, et d'une deuxieme période, provoquée par le décés d'un des conjoints, où le conjoint survivant est seul; la famille sera dissoute au monent du remariage du conjoint survivant ou de son déces en état de veuvage.

Ce schéma théorique amene deux commentaires:

- il est suffisamment genéral pour servir de guide dans lanalyse de situations où l'intensité des phénonenes démographiques qui président au début et a la fin des phases du cycle est tress différente;

- il est suffisamment souple pour permettre la comparaison fondé sur des sources d'information de nature differente, mais assez précis pour que des points de repere communs aux deux epoques puissent etre identifiés et utilisés de façon significative dans la comparaison. 
Figure 1 - Phases théoriques du cycle de la vie familiale

Événements démographiques marquant la vie des:

PHASES

1- PRE-PARENTALE

2- PARENTALE

a) agrandissement/contraction

b) stabilisation/contraction

3-POST-PARENTALE

a) conjointe

b) isolée

\section{PARENTS}
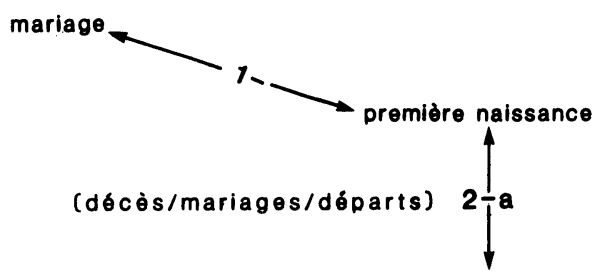

dernière naissance

(décès/mariages/départs)

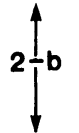

mariage/départ du dernier enfant

décès du deuxième conjoint

décès du premier conjoint : rupture d'union

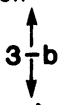

ou son remariage: dissolution

\section{COMPARAISON DU CYCLE DE VIE DEB FAMILLEB QUÉBÉCOI8E8 \\ AU XVIIE ET AU XXE SIÈCLES}

Pour mener la comparaison entre le cycle de vie des familles du XVIIe siecle et celles de la periode 1936-1955, deux sources seront utilisés.

Pour le XVIIe siecle, les données proviennent d'un échantillon de familles reconstituees automatiquement par le programe de recherche en demographie historique (P.R.D.H.) (Legaré, l981) pour les fins de trois monographies paroissiales: Beauport (Tremblay, 1981), Neuville (Bernier-Lemire, 1982) et la région Cap-santé - Deschamault - Grondines (Goudreau, 1984). Ces donnés portent sur les unions fondés avant 1700 observés jusqu'a la fin du régime français.

Cet echantillon, bien que petit, permet d'amorcer la recherche sur le cycle de la vie familiale au XVIIe siecle, en particulier par la mise au point de concepts et de methodes; de cette démarche, nous tirons des indications 
suffisamment fiables sur le déroulement de ce cycle pour les comparer au XXe siecle. Bien entendu, la poursuite de la recherche sur des nombres plus grands s'impose; elle est déjà en voie de réalisation'.

Voici les caracteristiques des familles retenues et exclues pour les diverses fins de l'analyse comparative. Au point de depart, l'échantillon se compose de 145 familles stables dont au moins les trois quarts des événements démographiques ont été enregistrés dans les paroisses rurales étudiés et dont les dates de début et de fin d'union sont connues. De ce groupe, on retient 74 familles où les deux conjoints se sont mariés célibataires et ont survécu jusqua la fin de la vie fertile de la femme, soit jusqua ce que celle-ci atteigne l'age de 45 ans. Etant donné le petit nombre de cas, les résultats devront etre interprétés avec prudence.

Pour les comparer au XXe siècle, on a recours aux données de l'enquete sur la fecondité des Québecoises ${ }^{2}$ et a un échantillon des familles tiré du recensement du Canadas; dans les deux cas, la collecte a eul lieu en 1971. Pour cette étude, ont eté sélectionnées les unions formées entre 1936 et 1955 , où les deux conjoints étaient célibataires et qui ne se sont pas rompues avant 1971. En annexe, on trouvera plus de détails sur ces données ainsi qu'une analyse montrant que les deux sources sont bien représentatives du mene univers.

Les données anciennes et modernes ne sont pas de meme nature, les unes se fondant sur une observation continue faite principalement a partir des actes d'état civil, les autres sur une observation rétrospective faite a un moment donné; dans les deux cas, les évenements relatifs a une meme famille peuvent etre rapprochés et analysés de façon longitudinale. Dans le premier cas, toutes les fanilles ont complété l'ensemble du cycle de vie; dans le second, le

1 La longue durée requise par la perspective longitudinale excluait l'utilisation de l'ensemble du fichier de familles reconstitués par le P.R.D.H. pour la période antérieure a 1730. Toutefois, dans la foulée de cette premiere etude exploratoire, le P.R.D.H. a deja entrepris l'analyse du cycle de vie des quelque 2500 familles pionnieres formés avant 1680 . Les résultats de cette étude en cours seront connus en 1985.

2 Enquete réalisée sous la direction de J. Henripin du Département de démographie de l'Universite de Montréal et subventionnée par le programme de subvention en bien-etre du ministere de la Santé et du Bien-être du Canada et par le ministere des Affaires sociales du Québec.

3 Echantillons au centieme des individus et des familles tirés du Recensevent du Canada 1971 et disponibles sur bande magnétique. 
cycle de la vie familiale est reconstitue a partir d un point donné qui tronque son déroulement. Toutefois, toutes les unions retenues ont atteint en 1971 une durée suffisamment longue (entre 15 et 34 ans) pour que 1 a periode correspondant a la vie feconde des femmes soit couverte; c est donc de façon longitudinale qu'on observe les phases pré-parentale et dagrandissement: ces données sont tout a fait comparables a celles du XVIIe siecle. Pour la séquence de stabilisation/contraction, les donnés modernes sont tronquees et il est nécessaire de supposer que la répartition des couples selon la duree du mariage et le stade deja atteint en 1971 est identique a ce que l'on aurait observé en suivant ces couples d'année en annép on fait alors 1 hypothese que la coupe transversale reflete le comportement de la cohorte: la régularite des résultats obtenus nous y autorise. Finalement, le début de la phase post-parentale pourra etre observé; cependant, la nature des donnés ne permettra pas la mesure de sa durée.

\section{PHASE PRÉ-PARENTALE}

Selon le schéma présenté a la figure 1, le cycle de la vie familiale debute par le mariage, point de depart de la phase pre-parentale. Des le moment de leur formation, les familles fondees au XVIIe et au XXe siecles se différencient; en effet le tableau 1 montre quavant 1700 , pres de $20 \%$ des mariages étudiés unissaient des conjoints où au moins l'un d'entre eux était veuf; entre 1936 et $1955,92 \%$ des unions se produisent entre celibataires et, parmi les autres, on observe l'emergence, encore faible, du remariage de divorces inexistant au XVIIe siecle. L'augmentation de l'importance relative des mariages entre celibataires tient sans doute au recul de la mortalité, phénomene qui autrefois provoquait de nombreuses ruptures d'union prématurés.

Les mariages d'autrefois et d'aujourd'hui se distinguent non seulement par l etat matrimonial des conjoints, mais aussi par l'age au mariage qui s'est considerablement modifié chez les femmes. En effet, on observe au tableau 2 que ces dernieres se mariaient environ 4 ans plus jeunes au XVIIe siecle quau cours de la période 1936-1955. Ce phénomene s'explique probablement par le désequilibre des sexes au début de la colonie où les femmes d'age nubile etaient rares. Comme l'gge au mariage a peu varié chez les hommes, une réduction de l'ecart d'age entre les conjoints s'en est suivie; il est passé de 7 a 3 ans. Notons de plus que l'age median n'est pas sensible au poids que representent les remariages dans l'ensemble des mariages; les remariages se produisent un age plus avance et l'age moyen reflete leur presence; il est en effet plus elevé pour l'ensenble des mariages que pour ceux des seuls célibataires: d'environ un an au XVIIe siecle, de presque deux ans au $x \times e$.

Le début du cycle de la vie familiale se caracterisait donc au XVIIe siecle par la formation d'unions ou les remariages etaient relativement nombreux et où la femme etait beaucoup plus jeune que son conjoint, meme dans les mariages entre celibataires. Par contre le milieu du Xxe siecle se distingue par une faible proportion de remariages et un ecart dige entre conjoints réduit. 
TABLEAU 1

Mariages suivant l'etat matrimonial antérieur des conjoints, Quebec, cohortes du XVIIe siecle et de la période 1936-1955

$\begin{array}{lcrr}\text { Epoux } & \text { Cohortes du XVIIe siecle } & (N=145) \\ & \text { Célibataire } & \begin{array}{c}\text { Vouse } \\ \text { Veuve }\end{array} & \text { Ensenble } \\ \text { Célibataire } & 81,4 & 10,3 & 91,7 \\ \text { Veuf } & 5,5 & 2,8 & 8,3 \\ \text { Ensemble } & 86,9 & 13,1 & 100,0\end{array}$

Epoux

Cohortes de la période $1936-1955 \quad(N=511674)$

Epouse

Célibataire Veuve Divorcé Ensenble

$\begin{array}{lrrrr}\text { Célibataire } & 91,9 & 1,3 & 0,3 & 93,5 \\ \text { Veuf } & 3,6 & 2,2 & 0,1 & 5,9 \\ \text { Divorcé } & 0,4 & 0,1 & 0,1 & 0,6 \\ \text { Ensemble } & 95,9 & 3,6 & 0,5 & 100,0\end{array}$

Sources: donnés anciennes: P.R.D.H.

données modernes: Bureau fédéral de la statistique,

Etat civil, 1936-1951.

TABLEAU 2

Age médian et moyen au mariage, Québec, cohortes du XVIIe siecle et de la période 1936-1955

$\begin{aligned} & \text { Cohortes du XVIIe siecle } \\ & \text { Mariages entre célibataires }\end{aligned}$
$\begin{aligned} & \text { Ensemble } \\ & \text { Nohortes } 1936-1955\end{aligned}$


Dans le déroulement du cycle de la vie familiale, le mariage marque lentrée en phase pré-parentale. En n'examinant désormais que les unions conclues entre célibataires et non rompues avant la fin de la période de fécondité de la femme, on constate que la durée de cette phase, qui se termine avec la venue du premier enfant, ne s'est a peu pres pas modifiée entre les deux époques; la durée médiane est resté à environ 1 an (tableau 3 ). De la meme façon, les conceptions prénuptiales restent exceptionnelles: environ $6 \%$ dans les deux cas.

En bref, il faut retenir qu'en depit de l'eloignement dans le temps, la phase pré-parentale a manifesté une grande stabilité; ce résultat, a premiere vue surprenant, montre bien que la régulation de la fécondité ne constituait pas encore une préccupation des jeunes couples formés avant 1956; en cela, les unions de la premiere moitié du xxe siecle se sont comportés tout a fait comme celles de l'epoque du régime français.

\section{PHABE PARENTALE}

La venue du premier enfant, marquant le debut de la phase parentale, represente le point de depart d'une longue periode dans la vie des couples dont la durée, d'ailleurs, ne semble pas s'etre modifiée considérablement: elle passe de 40 a 36 ans (tableau 4). Selon notre schéma, cette période se scinde en deux parties: la séquence d'agrandissement/contraction et celle de stabilisation/contraction.

La séquence d'agrandissement correspond à la période de constitution de la descendance. Entre les deux époques, on observe une transformation radicale de l'intensité de la fécondité: au xvile siecle, les fanilles completes avaient en moyenne 10,6 enfants; les familles formés entre 1936 et 1955 en ont eu 3 fois moins, soit 3,5 .

Examinons maintenant 1 'effet de cette reduction de la descendance sur la durée de la phase d'agrandissement: entre les unions du XVIIe siecle et les unions modernes, la duree tombe de plus de $50 \%$, passant de 21 ans a 9 ans. Il s'agit sans doute d'un des phénomenes dont les consequences sur la vie des individus et des familles sont les plus importantes; la vie des femmes en particulier en est completement bouleversée.

La différence d'intensite de la fecondite s'accompagne de conditions de mortalité des enfants tout aussi contrastés; la forte mortalité de jadis affecte le cycle de la vie familiale, car elle provoque rapidement la contraction des familles alors meme que la periode d'agrandissement n'est pas encore terminée. Au $X X e$ siecle, la situation est tout a fait différente: la fanille s'agrandit pendant une dizaine d'années puis reste stable pendant 14 ans; peu de familles sont touchées par le déces en bas age d'un de leurs enfants et le contraction ne se produit en genéral qu'au moment du départ des enfants. Le tableau 3 et la figure $2 a$ montrent a l'évidence le chevauchement de la phase dagrandissement et de contraction au XVIle siecle: quand $50 \%$ des familles arrivent a la stabilisation, soit apres 22 ans de mariage, déja plus de $90 \%$ ont connu une contraction, soit par le deces d un enfant, soit par un mariage. 
Durée médiane ${ }^{1}$ en années entre le premier mariage et le début des diverses phases du cycle de la vie

familiale, Québec, cohortes du XVIIe siecle et de la periode 1936-1955

\section{Durée médiane} entre le premier mariage et

Cohortes du
XVIIe siécle
$(N=74)$

$(N=74)$
Cohortes de $1936-1955^{3}$

$(N=399$ pour 1 et 2 ; $N=4596$ pour 3 et 41

1- la premiere naissance

2- la derniere naissance

3- le décès ou départ du premier enfant

(a) 135

(b) $6^{5}$

4- le décés ou départ

(a) 435 du dernier enfant

(b) 415

Sources: données anciennes: P.R.D.H.

donnés modernes: -Enquete sur la fécondité des Québécoises, 1971. -Echantillon au centieme des individus et des familles, Recensenent du Canada, 1971.

2 Voir en annexe les marges de variations lies a la taille des échantillons.

2 Mariages celibataire-célibataire, familles completes.

- Mariages célibataire-célibataire, familles où la durée de mariage est suffisamment longue pour englober la période de fécondité de la femme.

- Valeur estimée d'apres l'histoire des cohortes immediatement anterieures.

- Etant donné la proportion non négligeable d'enfants de destin inconnu (dont on ne connait que la date de naissance), on a dú etablir deux hypotheses de mortalité, l'une optimiste (a), l'autre pessimiste (b). L'hypothese optimiste (a) suppose que ces enfants ont survécu jusqu'd l'age médian au mariage, soit 26,2 ans pour les garsons et 18,9 ans pour les filles. L'hypothese pessimiste (b) suppose que ces enfants sont décedés le jour meme de leur naissance. L'hypothèse pessimiste (b) est vraisemblablement plus réaliste que l'hypothèse optimiste (a), car il est raisonnable de penser que la qualite de l'enregistrement des déces d'enfants a dú augmenter avec l'age. Par ailleurs, on a ramené a l'age médian au mariage le moment de depart des enfants mariés ou décédés comme célibataires au delà de cet age. 
Figure 2- PROPORTION DES FAMILLES QUI ONT DÉJÀ ATTEINT CERTAINES PHASES DU CYCLE DE LA VIE FAMILIALE

a) QUÉBEC, COHORTES DU XVIIe SIĖCLE

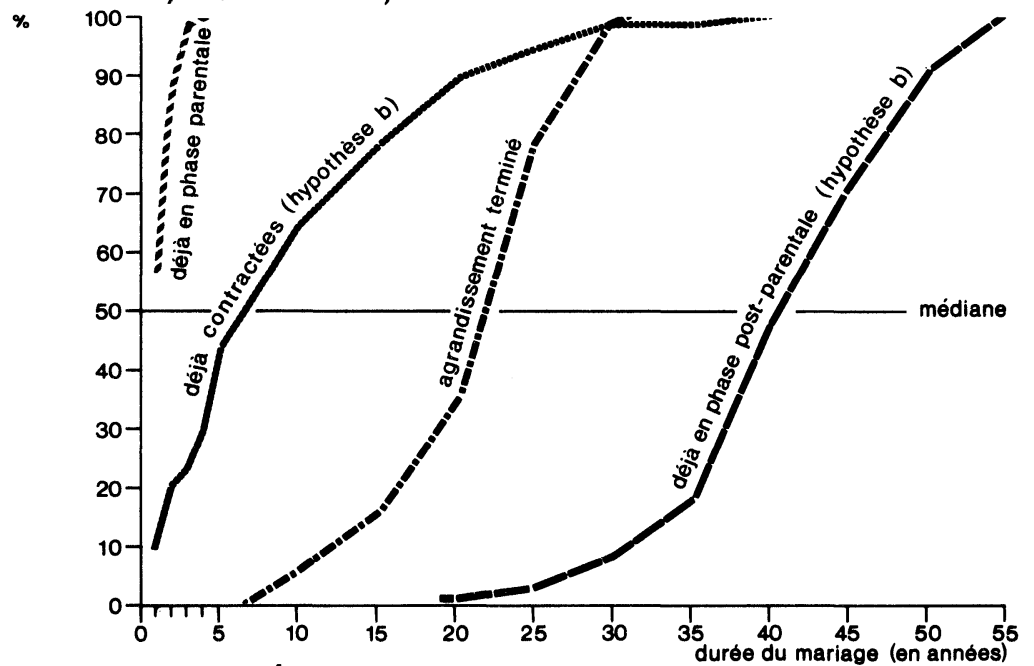

b) QUÉBEC, COHORTES DE 1936 - 1955

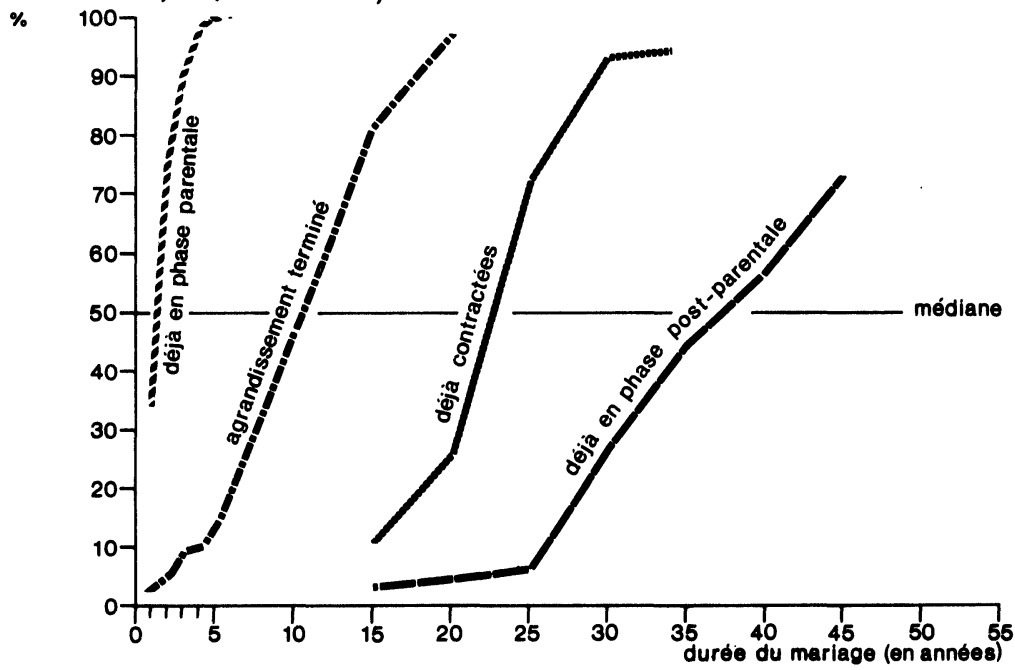

Sources: voir tableau 3 
Durée mediane en annees de certaines phases du cycle de la vie familiale, Québec, cohortes du XVIIe siecle et de la periode 1936-1955

\begin{tabular}{|c|c|c|}
\hline Phases & $\begin{array}{l}\text { Cohortes du } \\
\text { XVIIe siecle }\end{array}$ & $\begin{array}{c}\text { Cohortes de } \\
1936-1955\end{array}$ \\
\hline \multicolumn{3}{|c|}{ 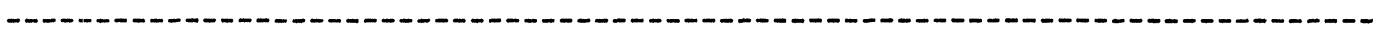 } \\
\hline Phase pré-parentale & 1 & 1 \\
\hline Phase parentale & 40 & 36 \\
\hline - Agrandissement/contraction & 21 & 9 \\
\hline - Stabilisation/contraction & 19 & 27 \\
\hline
\end{tabular}

Sources: voir tableau 3.

A lepoque moderne (figure $2 b$ ), quand $90 \%$ des familles se sont déja stabilisees, moins de $20 \%$ ont vu un de leurs enfants mourir ou les quitter. Malgré l'imprécision de ces résultats, on peut affirmer qu'au XVIIe siecle seule une infime minorité de familles vivaient une veritable phase de stabilisation oú les parents avaient la responsabilité de tous les enfants qu'ils mettaient au monde; cette situation, d'abord explicable par la forte mortalite infantile, était sans doute accentuée par le prolongement de la phase dagrandissement, les enfants preniers-nes, les filles en particulier, ayant déja atteint l'age au mariage au moment ou la mere donnait naissance a ses derniers enfants. Enfin, notons quautrefois entre la naissance du dernier enfant et le moment où le couple se retrouvait seul, il s'écoulait environ 19 ans; cette période s'étendrait aujourd'hui sur prés de 27 ans. Ce resultat étonnant reflete sans doute au $X X e$ siecle le prolongement des etudes, surtout chez les jeunes gens, et le mariage plus tardif chez les jeunes femmes. Notons de plus qu'une partie de la différence s'explique peut-etre, come nous le verrons a la section suivante, par les contraintes liées a la nature des données.

\section{PHASE POST-PARENTALE}

Les linites des donnés utilisées pour le $x \times$ siecle ne permettent que l'observation du début de la phase post-parentale; sa durée devrait etre estimée par l'espérance de vie du couple aprés le départ des enfants.

Au début de la phase post-parentale, la durée du mariage est de 37 ans au $x x e$ siecle, de plus de 41 ans au XVIle (tableau 3 ). Ces résultats, comme on l'a déja mentionné, indiqueraient que la durée de la phase parentale ne s'est pas considérablement modifiée. Cependant, il faut souligner que la mesure repose sur des bases relativement fragiles, car il n'est pas possible d'adopter pour les deux époques une démarche identique pour classer les 
couples en phase post-parentale: pour le XVIIe siecle, c'est le monent du mariage du dernier enfant ou l'age médian au mariage, dans le cas ou l'un des enfants ne se marie pas, qui servent de criteres; pour le XXe siecle, c'est la présence ou l'absence d'enfant dans le foyer des parents qui détermine la situation de ces derniers; la présence d' "enfant" est établie selon la définition de la famille de recensement dans laquelle le titre d" "enfant" est attribue a toute personne qui se déclare fils ou fille non marié(e) du chef de fanille, quel que soit l'age; cause de l'imprécision relative a l'age des enfants, il a eté impossible d'appliquer aux données modernes le critere de l'age au mariage. La solution adoptée entraine une surestimation de la durée du mariage au début de la phase post-parentale; cependant, le fait de retenir comme indice la durée médiane minimise l'effet du prolongenent du séjour des enfants dans une minorite de familles. Par ailleurs, le choix d'un critere significatif pour identifier le moment de l'entrée en phase post-parentale constitue en soi un objet de recherche qui n'a pas pu etre aborde en profondeur a ce jour. Avec les données présentement disponibles, l'ecart observé entre le XVIIe et le XXe siecles semble correspondre a un minimum.

\section{VUE D'ENSEMBLE SUR LE DÉROULEMENT DU CYCLE DE LA VIE FAMILIALE}

L'analyse que nous venons de présenter illustre l'évolution du déroulement du cycle de la vie familiale pour les familles qui en traversaient toutes les phases. Cette évolution est marquée par:

- la relative stabilité de la phase pré-parentale où le comportenent malthusien en début de vie conjugale est a peine perceptible chez les cohortes de 1936-1955;

- la réduction considérable de la durée de la phase diarandissement liée a la tres forte baisse de la fécondité;

- la disparition du chevauchement "agrandissement-contraction"; 1 époque moderne, cette séquence est devenue pour l'immense majorité des familles celle de l'agrandissement, grace au recul de la mortalité des enfants; la periode de contraction s'est déplacée et coincide avec le départ des enfants qui atteignent 1 'age adulte;

- lapparition d'une longue phase de stabilisation rélle au cours de laquelle les parents ont charge de tous leurs enfants; cette situation est le fruit de la baisse de la mortalite des enfants combinée a la réduction de la descendance qui raccourcit la periode dagrandissement;

- l'allongement de la periode qui va de la fin de l'agrandissement jusquau départ du dernier enfant;

post-parentale.

- la relative stabilité du moment oú les familles entrent en phase 
Ces résultats, extrêmement révelateurs en eux-memes par toutes les transformations des modes de vie évoqués, laissent dans l'ombre un aspect particulierement important du déroulement de la vie de l'ensemble des fanilles: l'impact des ruptures d'unions par veuvage ou divorce. Four completer l'image, sans qu'il soit toutefois possible de présenter le déroulement comparé de la vie de ces familles rompues en plein cycle, il est intéressant, et cela rejoint les préoccupations de Uhlenberg (1969), destimer la proportion des familles anciennes et modernes où les deux membres du couple voyaient se dérouler l'ensemble du cycle. On trouve ainsi a la figure 3 lévolution comparée de la survie des familles aux deux époques. Malgré son importance encore marginale, le divorce atténue déja la stabilité que la famille aurait gagné grace au recul de la mortalité des adultes.

\section{Figure 3-PROPORTION DES UNIONS NON ROMPUES SELON LA DUREEE DU MARIAGE}

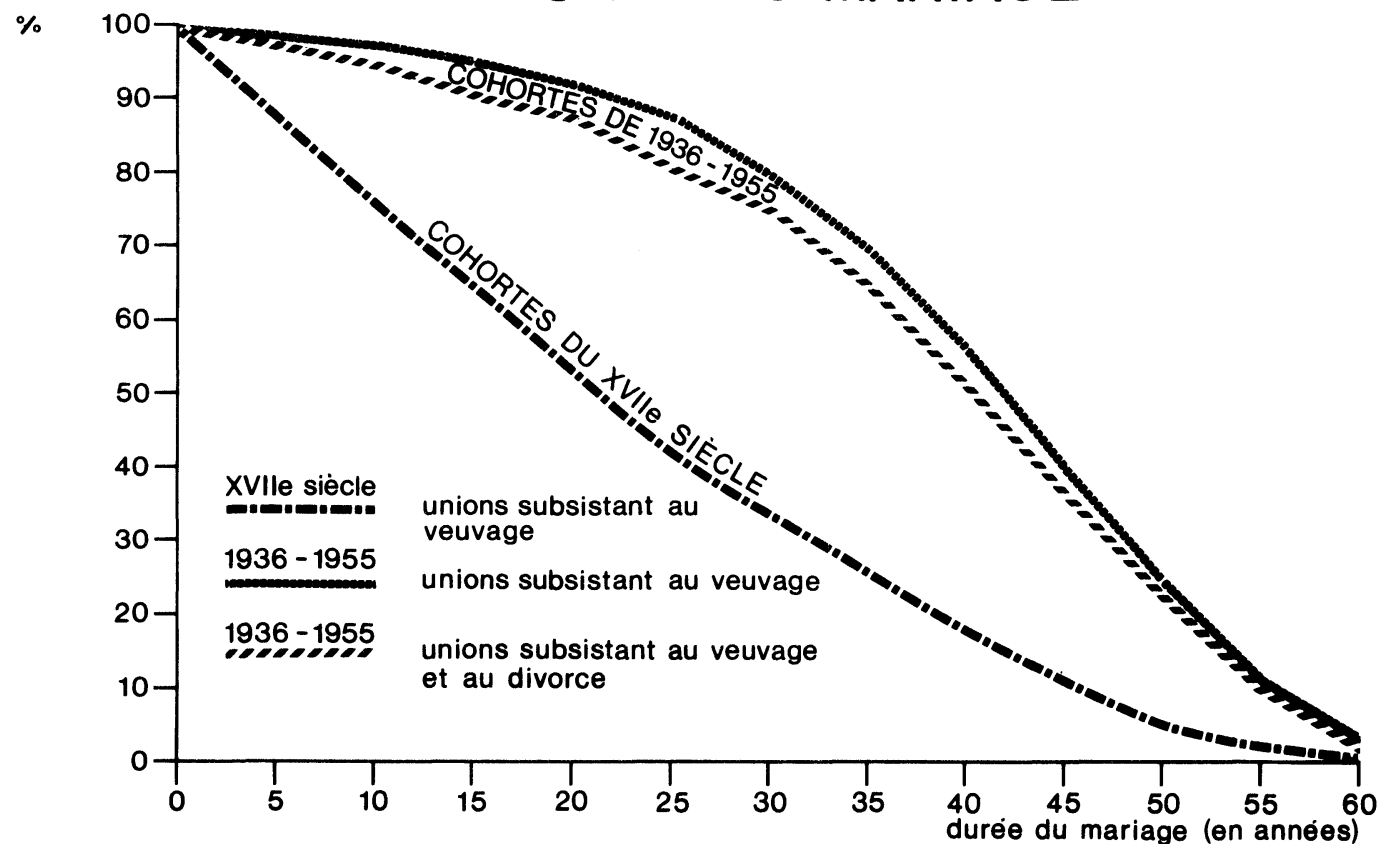

Sources: voir tableau 5

Les changements prennent une signification particuliere lorsqu'on rapproche ces courbes de survie des unions des durées medianes de mariage au debut ou a la fin des phases du cycle de la vie familiale (tableau 5 ); de ce rapprochement, on tire les observations suivantes:

- la grande majorité des unions formés entre 1936 et 1955 subsistent au moment où $50 \%$ des familles voient leur phase d'agrandissement se completer; 
Tableau 5

Durée médiane du mariage (en années) au moment de quelques phases du cycle de la vie familiale en 1 'absence d'événements perturbateurs et proportions correspondantes d'unions non rompues

\begin{tabular}{|c|c|c|c|c|}
\hline \multirow[b]{2}{*}{$\begin{array}{l}\text { Durée entre le } \\
\text { premier mariage et }\end{array}$} & \multirow{2}{*}{\multicolumn{2}{|c|}{$\begin{array}{l}\text { Cohortes du XVIIe } s . \\
\text { Durée } \% \text { diunions } \\
\text { mediane subsistantes" }\end{array}$}} & \multicolumn{2}{|c|}{ Cohortes de 1936-1955 } \\
\hline & & & $\begin{array}{l}\text { Durée } \\
\text { médiane }\end{array}$ & $\begin{array}{l}\% \text { dunions } \\
\text { subsistantes } 2\end{array}$ \\
\hline$-------------------m--$ & ------- & ------------- & $-----\cdots$ & ------------- \\
\hline - la premiere naissance & 1 & 97 & 1 & 99,8 \\
\hline -la dernière naissance & 22 & 46 & 10 & 94 \\
\hline $\begin{array}{l}\text { - le déces ou le départ } \\
\text { du premier enfant }\end{array}$ & $6^{3}$ & 86 & 24 & 85 \\
\hline $\begin{array}{l}\text { - le déces ou le départ } \\
\text { du dernier enfant }\end{array}$ & $41^{3}$ & 17 & 37 & 62 \\
\hline
\end{tabular}

Sources: voir tableau 3.

2 Table d'extinction des unions établie en fonction de l'experience de 477 mariages célébrés au Canada au XVIIe siecle (calculs inedits a partir de l'échantillon utilisé par Charbonneau, 1975).

Table dextinction des unions etablie en fonction des conditions de mortalite prévalant en 1951 et sous l'hypothese d'une fréquence de divorce égale a $7 \%$.

Hypothese de mortalité des enfants pessimiste.

plus de la moitié n'atteignaient pas cette duré au XVIIe siecle; si la descendance etait resté ce qu'elle était a cette epoque, la comparaison resterait largenent favorable a la période récente, car plus de $80 \%$ des familles subsistent toujours apres 22 ans de mariage;

- a l'epoque moderne, plus de $60 \%$ des couples subsistent encore a la duree où la phase post-parentale normalement commence; ce n'etait le cas autrefois que pour moins de $20 \%$ des couples; pour les familles du XVIIe siecle, connaître une période ou les deux conjoints se retrouvaient seuls apres avoir élevé leur fanille était une situation exceptionnelle; au XXe siecle, au contraire, c'est une solide majorité qui envisage de vieillir a deux; on peut parler de l'emergence d'une phase post-parentale qui s'est produite au cours de la longue période qui sépare les deux époques; 
- finalement, on observe de nouveau 1 impact de la réduction de la mortalite chez les enfants: l'epoque ancienne, la tres grande majorité des couples voyaient leur fanille se contracter prématurément, puisque $50 \%$ d'entre eux perdaient un enfant avant le sixiene anniversaire de mariagei l lepoque moderne, au $15 e$ anniversaire de mariage, environ $10 \%$ seulement des fanilles se sont contractés a cause d'un déces ou de l'absence d'un enfant qui vivrait en institution. Par ailleurs, pour le XVIIe siecle, si l'on ignore les contractions dues aux déces et que l'on suppose que le depart du premier enfant se produisait normalement vers le 24 e anniversaire de mariage des parents comme au Xxe siecle, environ $40 \%$ des unions subsistaient ce moment comparativenent a $85 \%$ a l'époque moderne.

\section{CONCLUSION}

En un peu plus de deux siecles, le cycle de vie des familles s'est transformé radicalement sous l'impulsion des changements dans les conditions de mortalité et de fecondité.

En l'absence d'évenements perturbateurs, mortalité des adultes et ruptures d'unions, c'est surtout la phase parentale qui distingue les deux époques: sa periode d'agrandissement qui s'étendait sur une vingtaine d'annés s'est réduite de plus de $50 \%$ sous l'impact de la reduction de la descendance; de plus, la simultaneité de l'agrandissement et de la contraction s'est estompee pour faire place a l'epoque moderne a une période de stabilisation d'environ 14 ans qui s'étend de la derniere naissance au départ du premier enfant; ce phénomene s'explique par le double effet de la réduction de la mortalité des enfants et de la fécondité.

Par ailleurs, les progres dus au recul de la mortalité chez les adultes ont entrainé une grande stabilité des unions de sorte que parmi les familles formés de 1936 a 1955 peu affectees par le divorce, la majorité traverseront sans veuvage l'ensemble des étapes du cycle de la vie familiale; ainsi 85\% d'entre elles subsistent toujours au moment où en général le prenier enfant quitte le foyer; ce n'était le cas que d'environ $40 \%$ au XVIle siecle; de plus, la fraction des familles qui arrivent a la phase post-parentale a plus que triple: seule une petite minorité y arrivaient autrefois; a l'epoque moderne, c'est le cas de la majorité.

Ces changements ont sans doute un impact considerable sur la vie des fanilles. Faut-il rappeler l'amelioration de la qualité de la vie liee au recul de la mortalite: la perte d'un enfant est devenue un événenent rare et le déces des parents se produit en général au moment ou tous les enfants ont atteint l'age adulte. Par ailleurs, la réduction de la descendance a allégé la responsabilité parentale et a sans doute profondément influence l'évolution des conditions dans lesquelles se deroule la vie des femmes en particulier.

On ne saurait terminer ces observations sans regretter que l'etat actuel de la recherche ne permette de présenter, meme a grands traits, les caractéristiques du cycle de vie des familles tout au cours des deux sidcles 
qui separent les périodes etudiées. Il serait intéressant de préciser a quelle époque les changements se sont produits, de determiner les périodes de stabilité et de dégager les mécanismes démographiques qui ont presidé au déroulement du cycle de la vie familiale. Le recul de la mortalité semblait devoir entrainer la famille dans un etat de stabilite. Ce ne sera sans doute pas le cas; déja le divorce, qui n'a touché que légerement les cohortes modernes etudiees, s'est accru de façon marquée pour les cohortes suivantes, au point qu'il menace les gains que la famille avait faits. La baisse de la fécondité s'est poursuivie et la descendance atteinte ne semble pas suffisante pour assurer le remplacement des génerations. Enfin, si le mariage constituait jusqua tout récemment le cadre dans lequel les couples voulaient vivre et constituer leur famille, de plus en plus de jeunes et de moins jeunes vivent maintenant ensemble sans etre mariés et ne jugent pas a propos de le faire, mene lors de la naissance de leurs enfants.

Ces nouveaux bouleversements dont les causes et les consequences sont mal connues représentent une justification de plus pour la poursuite des recherches sur l'evolution de la famille en géneral et du cycle de la vie familiale en particulier. Tout a fait appropries pour l étude du passé, les concepts et les mesures proposés ici devront sans doute etre adaptes pour tenir compte des situations nouvelles; toutefois, il ne faut pas écarter trop vite un outil qui permet d'analyser un phenomene en l'absence de perturbations, fournissant ainsi le point de repere a partir duquel l'importance rélle des transformations pourra etre evalué. Bien entendu, il reste fondamental, ainsi que nous l'avons fait, de situer dans le contexte des evenements perturbateurs, mortalité et divorce, le déroulement "normal" du cycle de la vie familiale.

Pour terminer, rappelons que la comparaison des familles du xVIIe et du xxe siecles met en lumiere des ecarts tres marques; malgre la taille réduite des echantillons, surtout celui du XVIIe siecle, et certaines hypotheses que nous avons dû poser, il est tres probable que des etudes reposant sur de meilleures donnés ne nous ameneront pas a réviser substantiellement nos conclusions; il faut toutefois s'attendre a ce que les résultats soient précisés, même revus, dans la poursuite des travaux. 
ANNEXE

\section{Échantilion extrait de l'enquete sur la fecondite des Qubbecoises, 1971}

Ce sondage a rejoint un échantillon representatif des femmes québécoises déja mariées et agés de 15 a 64 ans en 1971. On retrouve chez 458 dentre elles les caractéristiques suivantes: mariage non rompu, union entre célibataires célébrée entre 1936 et 1955.

De ce groupe initial, on a exclu pour les fins de l'analyse du cycle de la vie familiale les femmes suivantes:

- 38 femmes demeurées infécondes;

- 21 femmes pour lesquelles l'information relative aux dates de naissance des enfants est incomplete.

L'analyse porte donc sur 399 femmes fecondes; pour 1 'estimation de la durée de la phase préparentale, 22 femmes dont l'intervalle protogénésique est de plus de 60 mois ont eté exclues, en concordance avec la démarche adoptée pour le XVIIe siécle.

Comparason des echantillons tires de lienquete sur la fecondite et du recensement du Canada de 1971

A partir des deux sources, on a isole les femmes mariées entre 1936 et 1955 dont le mariage ne s'est pas rompu avant le moment de lobservation; seuls les mariages entre célibataires ont été retenus. On a comparé pour les deux groupes la distribution selon la durée du mariage et selon l'age.

Répartition en \% des femmes retenues suivant la durée du mariage d'apres deux sources

\begin{tabular}{llr}
$\begin{array}{l}\text { Durée du } \\
\text { mariage }\end{array}$ & $\begin{array}{l}\text { Recensement } \\
\text { du Canada }\end{array}$ & $\begin{array}{c}\text { Enquete sur } \\
\text { la feconditéz }\end{array}$ \\
\hline $15-19$ & 30,8 & 29,0 \\
$20-24$ & 28,0 & 28,7 \\
$25-29$ & 23,6 & 25,6 \\
$30-34$ & 17,6 & 16,8 \\
\hline Total & 100,0 & 100,0 \\
$N$ & 4596 & 458
\end{tabular}

Echantillon au centieme des familles, Recensement du Canada, 1971.

2 Enquete sur la fecondité des Québecoises, 1971. 


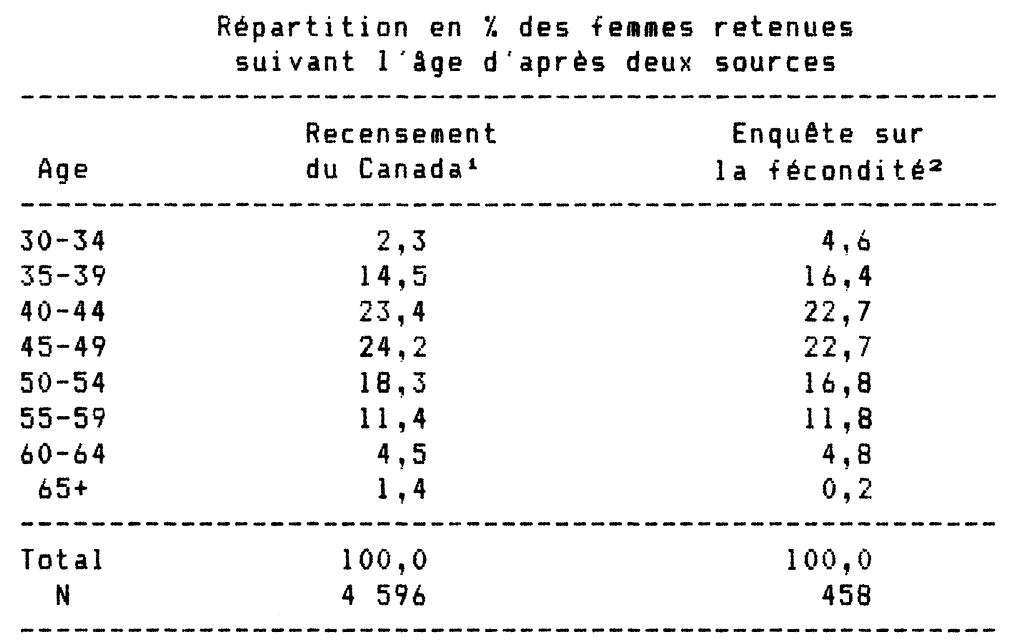

Echantillon au centieme des familles, Recensement du Canada, 1971. Enquête sur la fécondité des Québécoises, 1971.

Dans les deux cas, les valeurs obtenues pour le chi carré $\left(x_{3}^{2}=1,45\right.$ pour la durée du mariage, $x_{7}^{2}=5,69$ pour 1 'gge) ne sont pas statistiquement significatives (seuil de 0,05 ). Les deux echantillons representent donc le mene univers.

\section{Marges de variations des durees medianes lides a la taille des chantillons}

Niveau de signification: $95 \%$

Intervalle de confiance: $N=74, .50 \pm .11$

$N=399, \quad .50 \pm .05$

Intervalle en années a l'intérieur duquel devrait se situer la médiane

\begin{tabular}{lcc}
$\begin{array}{l}\text { Durée médiane } \\
\text { entre le mariage et }\end{array}$ & $\begin{array}{c}\text { Cohortes du } \\
\text { XVIIe siecle }\end{array}$ & $\begin{array}{c}\text { Cohortes de } \\
1936-1955\end{array}$ \\
\hline - la premiere naissance & 0,8 a 1,1 & 1,1 a 1,4 \\
- la derniere naissance & 20 a 23 & 10 a 11 \\
- le déces ou depart & 9 a 17 & 23 a 24 \\
du premier enfant & 5 a 9 & 35 a 37 \\
- le déces ou depart & 41 a 45 & \\
du dernier enfant & 39 a 43 &
\end{tabular}




\section{RÉFÉRENCES BIBLIOGRAPHIQUES}

BERNIER-LEMIRE, Lorraine, 1982. Neuville, des origines a 1762: etude déographique d'une paroisse rurale du Quebec. Mémoire de mâtrise, Département de demographie, Université de Montréal, $196 \mathrm{p}$.

CHARBONNEAU, Hubert, 1975. Vie et mort de nos ancetres. Etude denographique. Montréal, Les Presses de l'Université de Montréal, 267 p. (Coll. "Démographie canadienne", no 3 ).

FoURAStIE, Jean, 1959. "De la vie traditionnelle a la vie tertiaire". Population, 14,3, 417-432.

GLICK, Paul C., et PARKE, Robert, Jr., 1965. "New Approaches in Studying the Life of the Family". Denography, 2, 187-202.

GOUDREAU, Serge, 1984. La population de Cap-Santé, de Deschabault et de Grondines sous le régine frangais (1679-1762). Memoire de maitrise, Département de démographie, Université de Montréal, 205 p.

LANDRY, Yves et Legare, Jacques, 1984. "Le cycle de vie familiale en Nouvelle-France: méthodologie et application a un échantillon". Histoire sociale/Social History, XVII, 33, 7-20.

LEgARE, Jacques, 1981. "Le Programme de recherche en démographie historique de 1'Université de Montréal: fondements, méthodes, moyens et résultats". Etudes canadiennes/Canadian Studies, 10, 149-182.

LIVI-BACCI, Massimo, 1978. "Le changement démographique et le cycle de vie des femmes", in Evelyne Sullerot, Le fait feninin. Paris, Fayard, 467-478.

PERON, Yves et LAPIERRE-ADAMCYK, Evelyne, 1984. "Structures familiales et calendrier démographique familial: un essai d'application a la situation des familles du Quebec". Rapport final, Prograne de recherche cooperative inter-centres. Paris, CICRED, 136-139.

TREMBLAY, Micheline, 1981. La population de Beauport sous le regine francais. Memoire de maitrise, Département de démographie, Universite de Montréal, $320 \mathrm{p}$.

UHLENBERG, Peter R., 1969. "A Study of Cohort Life Cycles: Cohorts of Native Born Massachussets Women, 1830-1920". Population Studies, 23, 3, 407-420. 


\section{RÉSUME - SUMHARY - REBUMEN}

LAPIERRE-ADAMCYK Évelyne, LANDRY Yves, LÉgaré Jacques, MORISSEtTE denis et PÉroN Yves - Le cyele de la vie fanillale au Qubbeci vues conparatives, XVIle - Xxo slicles

Cet article vise à conparer le cycle de la vie faniliale tel qu'on a pu l'observer au Québec a deux époques éloignées l'une de l'autre: la fin du XVIle siécle et le ailieu du XXe siecle. Le calcul d'indices comparables a été réalisé pour les deux périodes en l'absence d'événenents perturbateurs. Il pernet de faire ressortir les traits arquants de l'évolution du cycle de la vie faniliale pour les fanilles qui en traversaient toutes les phases; soulignons en particulier la réduction considérable de la durée de la phase d'agrandissenent, liée a la très forte baisse de la fécondité, et l'apparition d'une longue phase de stabilisation réelle au cours de laquelle les parents ont charge de tous leurs enfants. Un rapprochement avec la proportion des fanilles que représentent les unions non roupues laisse enfin apparaitre de façon éclatante l'iapact de la nortalité sur la vie faniliale d'autrefois.

LAPIERRE-ADAMCYK Évelyne, LANDRY Yves, LÉgaré Jacques, MORISSETte Denis and PÉRON Yves - The Fanily Life cycle in Qubbeci Comparative Views, 17th - 20th centuries

The ain of this article is to conpare the fanily life cycle as observed in Quebec for two distant time periods: the end of the 17th century and the niddle of the 20th century. For both periods, calculations of comparative indices mere carried out in the absence of oortality and divorce. This allows the eaphasizing of the outstanding features of the fanily life cycle, for fanilies having experienced all the phases; let us underline, in particular, the considerable reduction in the duration of the extensive phase, which is linked to the significant drop in fertility, and the energing of a long phase of stabilization during which the parents are responsible for all their children. Finally, an exanination of the proportion of undissolved fanilies at each stage clearly shows the dranatic inpact of mortality on family life in the past.

LAPIERRE-ADAMCYK Évelyne, LANDRY Yves, LÉGARÉ Jacques, MORISSETte denis y PÉRON Yves - El eiclo de vida de las fanillias de Quebecs exanen conparativo, siglos XVII y XX

El presente artículo está encaninado a comparar el ciclo de vida de las fanilias de Quebec en dos épocas alejadas una de la otra para las que fue posible hacerlo: a fines del siglo xVII y a mediados del $X X$. En anbos periodos, el cálculo de los indices comparativos se efectuó en ausencia de fenómenos perturbadores. Este hecho pernite poner de relieve las caracteristicas deterninantes de la evolución del ciclo de vida faniliar para las fanilias que han atravesado por cada una de las etapas de este ciclo. Hay que hacer notar, particularaente, la reducción considerable de la duración de la fase de creciniento, relacionada con el brusco descenso de la fecundidad y la aparición de une fase prolongada de estabilización real, durante la cual los padres tienen a su cargo a todos los hi jos. Por últino, tomando en cuenta únicanente la proporción de fanilias en las que la unión no ha sido interrunpida y su relación con diversos parámetros, se aprecia claranente el inpacto de la mortalidad sobre la vida faniliar de antaño. 